\section{THU0582 A SYSTEMATIC REVIEW OF EMPLOYMENT OUTCOMES OF ADULTS WITH CHILDHOOD-ONSET SYSTEMIC AUTOIMMUNE RHEUMATIC DISEASES}

\section{K. Gu, T. Gottschalk, L.S.H. Lim. University of Manitoba, Winnipeg, Canada}

Background: Childhood-onset systemic autoimmune rheumatic diseases (ChildCRD) include: systemic lupus erythematosus (SLE), Sjogren's syndrome, systemic sclerosis, inflammatory myositis, and chronic systemic vasculitides (Takayasu arteritis, polyarteritis nodosa, anti-neutrophil cytoplasmic antibodies (ANCA) associated systemic vasculitides). These conditions had mortality rates of up to $50 \%$, but most patients now live into adulthood. Employment is an important milestone in adulthood: it has direct effects on one's socioeconomic status and access to health insurance, which could in turn affect disease outcomes.

Objectives: To perform a systematic review of the employment outcomes of ChildCRD individuals. To identify gaps of knowledge and methodological issues in this field so as to inform future studies.

Methods: ChildCRD patients have disease-onset $<18$ years old and adulthood outcomes reported at $\geq 18$ years old. We developed a search strategy for employment outcomes of ChildCRD with an academic librarian; this was iteratively refined and finalised after peer-review by other librarians. We included English language articles published from Jan 1990 Oct 2017 in MEDLINE, EMBASE, and Scopus. Case reports, case series, editorials, letters, or short reports were excluded. We supplemented our search by hand-searching references in review articles. Information on outcomes, prognostic factors, and study designs was recorded. Studies were graded independently by 2 reviewers (after prior training for agreement) using the Quality in Prognosis Studies (QUIPS) risk-of-bias tool which examines quality in 6 study domains. Authors were contacted as necessary for further information or clarification.

Results: Of 2109 studies, we identified 3 publications (G1) studying SLE patients. None studied other ChildCRD. Two papers were from a single study and studied both SLE and juvenile arthritis; we only used SLE patients' information. Three additional manuscripts (G2) studied childhood- and adult-onset patients but did not report outcomes separately. All G1 publications were from North America (2 Canada, 1 USA). 193 patients in 2 studies were examined; 1 study had longitudinal (non-inception) design. Only G1 studies have data for report. Patients' disease durations were a mean of 7.6-15 years and the mean ages at study were 23-29 years. Half of the patients were employed. Lower disease activity, better physical function, and higher education were associated with longitudinal employment in 1 study; greater independence and social support were associated with employment in another. One study showed psychosocial and work context factors to be associated with absenteeism, disease activity with job disruptions, and fatigue and work context factors with lost productivity. Of QUIPS-graded G1 publications, study populations and confounding were at moderate-high risk-of-bias. Study outcomes, prognostic factors, and statistical analyses were at low-moderate risks-ofbias. Attrition was low in the longitudinal study.

Conclusions: Currently, there is minimal information on employment outcomes in ChildCRD adults except for few studies on SLE; information about other ChildCRD is needed. Study populations and confounding are at moderate-high risks-of-bias, limiting the generalizability. More information on employment outcomes, the specific aspects of employment, disease and non-disease related prognostic factors affecting employment are needed.

Disclosure of Interest: None declared

DOI: 10.1136/annrheumdis-2018-eular.4347

\section{THU0583 PAIN INTERFERENCE ASSOCIATED FACTORS IN A COHORT OF FINNISH YOUNG ADULTS WITH JUVENILE IDIOPATHIC ARTHRITIS}

K. Rebane ${ }^{1}$, T. Orenius ${ }^{2}$, L. Ristolainen ${ }^{2}$, H. Relas ${ }^{3}$, H. Kautiainen ${ }^{4}$, R. Luosujärvi ${ }^{3}$, H. Säilä ${ }^{2}$, K. Aalto ${ }^{1}{ }^{1}$ Children's Hospital, University of Helsinki and Helsinki University Central Hospital; ${ }^{2}$ Orton Ortopaedic Hospital, Orton; ${ }^{3}$ Rheumatology, Helsinki University Central Hospital; ${ }^{4}$ Department of General Practice and Primary Health Care, University of Helsinki and Helsinki University Central Hospital, Helsinki, Finland

Background: Pain is one of the most distressing and persisting features of JIA and frequently interferes with everyday life.

Objectives: This study was conducted to find out the factors associated with pain interference in young adults (aged 18 to 30 years) with JIA.

Methods: 195 adult patients with JIA were eligible for the study. Associations between patients reported outcome data and pain interference were examined. Sociodemographic and clinical data were analysed. Pain interference was measured by a single item from the RAND 36 questionnaire. Five response categories were coded into different groups: patients reporting "extremely" and "quite a bit" or "moderate" were classified having significant pain interference; "a little bit" as having minor pain interference; and "not at all" as having no pain interference. Functional disability was measured by $\mathrm{HAQ}$, depressive symptoms were measured by Beck Depression Inventory-II, self-esteem was assessed by Rosenberg Self-
Esteem Scale, and anxiety was assessed by PASS-20. Leisure time physical activity (LTPA) metabolic equivalent (MET) score was calculated.

Results: Pain intensity scores were higher in patients expressing significant pain interference (mean 5.3, SD 2.1) and minor pain interference (mean 2.8, SD 2.09); $p$ for linearity $<0.001$, thus the mean pain intensity for the whole study group was quite low (mean 2.3, SD 2.3). Of the 195 patients 98 (50.3\%) reported no pain interference, 59 (30.3\%) reported minor pain interference, and $39(20 \%)$ reported significant pain interference. We found that pain interference was associated with older age $(p=0.029)$, female gender $(p=0.028)$ and receiving antirheumatic treatment $(p=0.032)$, analgesics $(p<0.001)$, antidepressants $(p=0.008)$, and opioids $(p<0.001)$. Also cohabiting $(p=0.003)$, LTPA MET $(p=0.032)$, smoking $(0.006)$, being more disabled $(p<0.001)$, having fewer leisure time activities $(p<0.001)$ or having co-morbidities $(p=0.006)$, and headache $(p<0.001)$ were associated with having pain interference. Higher anxiety scores were associated with more pain interference $(p<0.001)$. When controlling for gender, age, depression, LTPA MET, disability, life situation, disease remission, analgesics, antidepressants, and pain intensity, all subscales in PASS-20 were significantly associated with higher pain interference levels: cognitive anxiety $(p=0.004)$, escape/avoidance $(p<0.001)$, fear $(p=0.001)$, psychological anxiety $(p=0.016)$.

Conclusions: Half of the JIA patients reported pain interference, and they also expressed higher pain scores. Age, gender, using antirheumathic drugs, antidepressants, analgesics or opioids, cohabiting, lower LTPA MET score, disability, smoking, co-morbidities, lack of activities, and suffering anxiety were most significantly related to pain interference. Our study highlights the need to develop bette strategies for pain-relieving interventions and for supporting patients' healthbehaviour in order to achieve better pain outcome in young adults with JIA.

Disclosure of Interest: None declared DOI: 10.1136/annrheumdis-2018-eular.5180

\section{THU0584 VITAMIN D STATUS IN PATIENTS WITH JUVENILE IDIOPATHIC ARTHRITIS AND ITS ASSOCIATION WITH DISEASE ACTIVITY AND PROGRESSION - RESULTS FROM THE INCEPTION COHORT ICON}

C. Sengler ${ }^{1}$, J. Zink ${ }^{1}$, J. Klotsche ${ }^{1}$, D. Foell ${ }^{2}$, M. Niewerth ${ }^{1}$, I. Liedmann ${ }^{1}$, G. Horneff ${ }^{3}$, J.-P. Haas ${ }^{4}$, K. Moenkemoeller ${ }^{5}$, A. Thon ${ }^{6}$, K. Minden ${ }^{1} .{ }^{1}$ Epidemiology unit, GERMAN RHEUMATISM RESEARCH CENTRE, Berlin; ${ }^{2}$ Department of Paediatric Rheumatology and Immunology, University of Muenster, Muenster, ${ }^{3}$ Asklepios Clinic Sankt Augustin, Sankt Augustin; ${ }^{4}$ German Centre for Paediatric and Adolescent Rheumatology, Garmisch-Partenkirchen, ${ }^{5}$ Clinics of the City of Cologne, Cologne; ${ }^{6}$ Hannover Medical School, Hannover, Germany

Background: Vitamin $\mathrm{D}$ has been shown to have immunomodulatory properties in addition to its well-established role in the maintenance of mineral homeostasis and bone health. Conflicting data have been available regarding vitamin $D$ status in children and adolescents with juvenile idiopathic arthritis (JIA) and its influence on the disease.

Objectives: To determine 25-0 hour-vitamin D3 (25-OHD) levels in patients with $\mathrm{JIA}$, and to evaluate whether $25-\mathrm{OHD}$ levels are associated with disease activity and disease course.

Methods: Serum 25-OHD levels were determined in a pair of serum samples from patients with JIA who were enrolled in the JIA inception cohort study ICON and prospectively followed for three years. The frequencies of 25-OHD deficiency (defined as $<20 \mathrm{ng} / \mathrm{ml}$ ) and 25-OHD sufficiency $(\geq 30 \mathrm{ng} / \mathrm{ml}$ ) were determined and compared with those of the age-, sex- and ethnicity-matched general population (KIGGS study). The association of 25-OHD levels and the likelihood for uveitis were analysed by a Cox-proportional hazard model. Logistic regression analysis was used to investigate the predictive value of $25-\mathrm{OHD}$ in terms of disease progression into extended oligoarthritis $(\mathrm{OA})$.

Results: In 360 patients with early JIA (48\% OA, 27\% rheumatoid-factor negative polyarthritis), 25-OHD levels were determined twice: after a median disease duration of 6.6 and of 13.0 months. The mean 25-OHD level of all JIA samples was insufficient $(22.1 \mathrm{ng} / \mathrm{ml}, \mathrm{SD} 7.8)$, but significantly higher than that of the control population $(18.4 \mathrm{ng} / \mathrm{ml}, \mathrm{SD} 10, \mathrm{p}<0.001)$. Vitamin D levels decreased with age in patients and controls, but did not differ between males and females

An insufficient mean $25-\mathrm{OHD}$ level was found in $40 \%$ of $\mathrm{JIA}$ patients and in $25 \%$ in both samples each, sufficient levels in $13 \%$ and $7 \%$, respectively. There were no significant differences in 25-OHD levels among the JIA categories. Disease activity, measured by the cJADAS-10, was inversely correlated with the first 25-OHD level $(\beta=-0.20,95 \% \mathrm{Cl}-0.37,-0.03, p=0.018)$, especially in 141 DMARD-naïve patients $(\beta=-0.26,95 \% \mathrm{Cl}-0.44,-0.01, p=0.041)$. Up to the 3 -year-follow-up, $17 \%(61 / 360)$ developed uveitis, and $30 \%(52 / 173)$ of OA patients an extended OA. While $20 \%(17 / 87)$ of those with 25-OHD deficiency at both measurements were affected by uveitis, this applied to only $9 \%(2 / 23)$ in those with sufficient levels. Multivariable regression analysis revealed that the 25-OHD level was significantly associated with the risk to develop uveitis (Hazard ratio $0.95,95 \% \mathrm{Cl}$ $0.91,0.99, p=0.008)$. Twelve out of $29(41 \%)$ patients with OA and two deficient 